\title{
Strain Analysis of a Disk Subjected to Diametral Compression by Means of Holographic Interferometry
}

\author{
Cesar A. Sciammarella and John A. Gilbert
}

\begin{abstract}
Two simultaneous holograms and several directions of observation are utilized to determine the components of displacement and strain in the disk. A reference strip is introduced to relate the fringe orders in the two holograms. A very good agreement is obtained between the holographic results and the elasticity theory solution. The obtained displacement field is estimated to be accurate to $8 \times 10^{-6} \mathrm{~cm}$, which is approximately one-eighth the wavelength of the laser light utilized to obtain the holograms.
\end{abstract}

\section{Introduction}

Holographic interferometry is a powerful tool when used to determine the displacement of surface points of arbitrarily shaped bodies.

The basic theoretical aspects of hologram interferometry as a means of displacement determination have been developed by the contributions of many different authors. Starting with the pioneering work of Powell and Stetson, ${ }^{1,2}$ further contributions have been made by Haines and Hildebrand, ${ }^{3}$ Aleksandrov and Bonch-Bruevich, ${ }^{4}$ Ennos, ${ }^{5}$ Sollid, ${ }^{6}$ Sampson, ${ }^{7}$ Stetson, ${ }^{8-10}$ Walles, ${ }^{11}$ Viènot et al., ${ }^{12,13}$ and Abramson. ${ }^{14-17}$

Applications to the general problem of obtaining more than one component of the displacement vector have been made among others by Ennos, ${ }^{5}$ Wilson, ${ }^{18}$, 19 and Shibayama and Uchiyama. ${ }^{20}$

The purpose of this paper is to apply the general equations of holographic interferometry to an elasticity problem of known solution in order to evaluate the experimental results.

The main objectives of the paper are (1) to develop general techniques that can be applied to problems that are not restricted by special features introduced to simplify the experimental analysis, (2) to find the limits of accuracy that can be achieved in the measurement of displacements using holographic interferometry, and (3) to provide an insight on further steps to be followed to make holographic interferometry a reliable and valuable tool in the field of optical stress analysis.

The authors are with the Experimental Mechanics Laboratory, Illinois Institute of Technology, Chicago, Illinois 60616.

Received 20 December 1972.
A disk under diametral compression was chosen as a model. This model, although simple to investigate from the point of view of other optical techniques, presents in the case of holographic interferometry some interesting features. The model is supported only in two small regions of the loaded diameter. This characteristic makes it very difficult to avoid rigid body motions that are not related to the displacements generated by the applied load.

Since holographic interferometry has a very high sensitivity, rigid body motion may exhaust all its capability of recording displacement information. That is, the analysis of the fringes will reveal mostly rigid body displacements. Furthermore, these rigid body motions will not be in general reproducible from load to load, thus preventing the possibility of taking successive holograms of the same object under the same load but with different directions of illumination and observation.

Since the observed fringe pattern of the disk does not show a zero-order fringe, it becomes necessary to establish a common datum point in order to correlate multiple holograms.

\section{Holographic Technique}

To solve the problem under analysis, we had the choice of applying the single-hologram technique or the multiple-hologram technique. In the present case, it was clear that the fringe shift that could be obtained using various directions of observation on a single hologram was not sufficient to obtain an accurate determination of the three-dimensional displacement field. A multiple-hologram technique offered the advantage of allowing us the optimization of the detection of the smaller components of the displacement field. However, to apply this technique it is necessary to have a common datum point for the different holograms. This was achieved by 


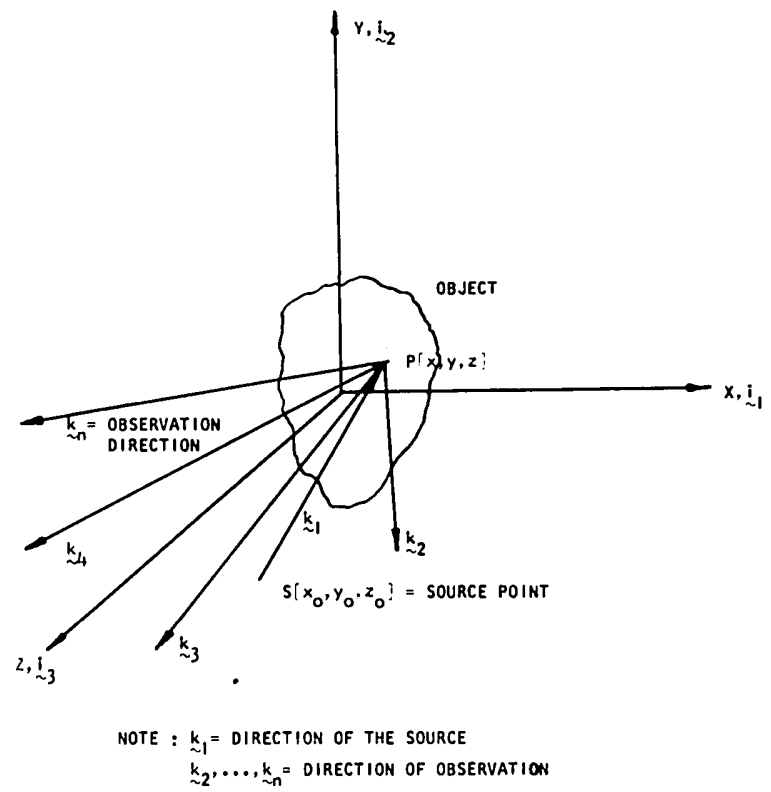

Fig. 1. The analysis of a surface point $P$.

introducing an elastic strip rigidly attached to the holographic table and to a point along the horizontal diameter of the disk. A slight initial tension was applied to the elastic rubber strip. The principal displacement experienced by the strip when the model is loaded is an elongation in the axial direction.

Let us consider now the coordinate system $x, y, z$, with corresponding unit vectors $\mathbf{i}_{1}, \mathbf{i}_{2}, \mathbf{i}_{3}$ (Fig. 1). The propagation vector from the source point $S\left(x_{0}, y_{0}, z_{0}\right)$ is denoted by $\mathbf{k}_{1}$, while the propagation vectors $\mathbf{k}_{j}, j=2,3, \ldots \eta$ correspond to the observation points $O\left(x_{j}, y_{j}, z_{j}\right)$, where $j=2,3, \ldots \eta$. The $\eta-$ 1 points of observation are situated on one or on several holograms. Point $P(x, y, z)$ is a point on the surface of the object whose displacement vector is to be determined.

We can write for the point $P^{6}$

$$
\delta_{j}=-\left(\mathbf{k}_{1}-\mathbf{k}_{j}\right) \cdot \mathrm{d}=2 \pi n_{j} \quad j=2,3, \ldots \eta,
$$

where $\delta_{j}$ is the phase difference for the $j$ direction of observation between the unloaded and loaded conditions, $\mathbf{k}_{1}=(2 \pi / \lambda) \hat{\mathbf{k}}_{1}, \mathbf{k}_{j}=(2 \pi / \lambda) \hat{\mathbf{k}}_{j}$, where the symbol ' indicates a unit vector, $\mathbf{d}$ is the displacement vector experienced by the point $P$ and $n_{j}$ is the fringe order.

For the coordinate system of Fig. 1,

and

$$
\mathbf{d}=u \mathbf{i}_{1}+v \mathbf{i}_{2}+w \mathbf{i}_{3}
$$

$$
\hat{\mathbf{k}}_{1}=\left[\left(x-x_{0}\right) / R_{0}\right] \mathrm{i}_{1}+\left[\left(y-y_{0}\right) / R_{0}\right] \mathrm{i}_{2}+\left[\left(z-z_{0}\right) / R_{0}\right] \mathbf{i}_{3}
$$

where

$$
R_{0}=\left[\left(x-x_{0}\right)^{2}+\left(y-y_{0}\right)^{2}+\left(z-z_{0}\right)^{2}\right]^{1 / 2}
$$

and similarly for $j=2,3 \ldots \eta$

$$
\hat{\mathbf{k}}_{j}=\left[\left(x_{j}-x\right) / R_{j}\right] \mathbf{i}_{1}+\left[\left(y_{j}-y\right) / R_{j}\right] \mathbf{i}_{2}+\left[\left(z_{j}-z\right) / R_{j}\right] \mathrm{i}_{3}
$$

and

$$
R_{j}=\left[\left(x_{j}-x\right)^{2}+\left(y_{j}-y\right)^{2}+\left(z_{j}-z\right)^{2}\right]^{1 / 2} .
$$

Replacing Eqs. (2), (3), and (5) in (1) and taking into consideration the definitions of $\hat{\mathbf{k}}_{0}$ and $\hat{\mathbf{k}}_{j}$, we obtain

$$
\begin{aligned}
& \left\{\left[\left(x_{j}-x\right) / R_{j}\right]+\left[\left(x_{0}-x\right) / R_{0}\right]\right\} u \\
& +\left\{\left[\left(y_{i}-y\right) / R_{j}\right]+\left[\left(y_{0}-y\right) / R_{0}\right]\right\} v+\left\{\left[\left(z_{j}-z\right) / R_{j}\right]\right. \\
& \left.+\left[\left(z_{0}-z\right) / R_{0}\right]\right\} w=n_{j} \lambda \quad j=2,3, \ldots \eta .
\end{aligned}
$$

We have a linear system of $\eta-1$ equations in the three unknowns $u, v, w$. To optimize the solution of the system, the least-squares method can be utilized. ${ }^{21}$ The above equations can be written

$$
F^{\prime}(u, v, w)=C_{j} \quad j=2,3, \ldots \eta .
$$

The system of $\eta-1$ equations in three unknowns can be reduced to a three-by-three set as follows:

$$
\begin{aligned}
& \frac{\partial}{\partial u}\left\{\sum_{j=2}^{n}\left[F_{j}(u, v, w)-C_{j}\right]^{2}\right\}=0, \\
& \frac{\partial}{\partial v}\left\{\sum_{j=2}^{n}\left[F_{j}(u, v, w)-C_{j}\right]^{2}\right\}=0, \\
& \frac{\partial}{\partial w}\left\{\sum_{j=2}^{n}\left[F_{j}(u, v, w)-C_{j}\right]^{2}\right\}=0 .
\end{aligned}
$$

\section{Experimental}

A $10.0-\mathrm{cm}$-diameter plexiglas disk $(E=28.12 \mathrm{~kg} /$ $\left.\mathrm{cm}^{2}, \nu=0.385\right)$ was subjected to diametral compression. The disk was painted flat white and a grid $12.51 \mathrm{~mm}$ square was scribed on the surface. A helium-neon laser having a power output of $80 \mathrm{~mW}$ and a wavelength $\lambda=63.28 \times 10^{-6} \mathrm{~cm}$ was used as a light source. The reference coordinate system was placed with its origin in the center of the disk. The $x$ and $y$ axes were located on the surface of the model

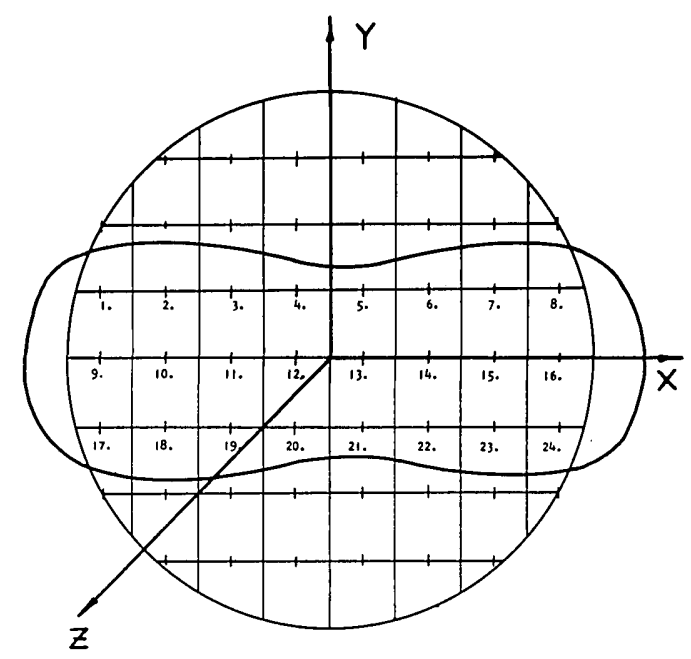

Fig. 2. Coordinate system and reference grid. 


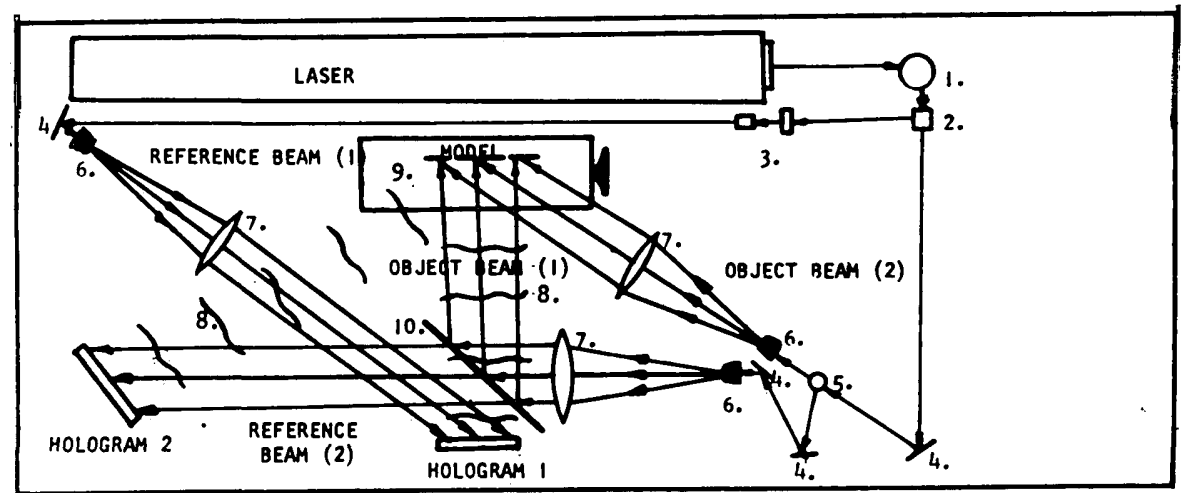

Fig. 3. Experimental setup.

\author{
I. HEIGHT ADJUSTER \\ 2. BEAM SPLITTER \\ 3. ADJUSTABLE POLAR ISCOPE \\ 4. MIRROR \\ 5. ADJUSTABLE. BEAM SPLITTER
}

\author{
6. SPATIAL FILTER \\ 7. LENS \\ 8. DIFFRACTEO OBJECT WAVEFRONT \\ 9. LOADING MECHANISM \\ 10. PARTIAL MIRROR
}

with the $y$ axis parallel to the direction of the applied load (vertical direction) and the $x$ and $z$ axes pointing in the directions indicated in Fig. 2. The experimental setup is sketched in Fig. 3.

Two holograms were made using two separate pairs of object and reference beams. After the blocking of object beam 2, the unloaded condition was recorded on holographic plate 1 using object beam 1 , generated by the partially reflecting mirror, and reference beam 1 . Object and reference beams 1 were now blocked and the unloaded state was then recorded in hologram 2 using object and reference beams 2 . The two separate exposures were necessary to avoid interference effects that would have been caused by two simultaneous object beams. The model was now loaded and this loaded condition was superimposed onto holograms 1 and 2 employing the same procedure as above. The aim of the over-all process was to ensure an exact duplication of load in the two separate holograms.

The location of the holographic plates was important. Hologram 1 was positioned to favor the deter- mination of $w$, the smallest of the three displacement components. That is, the normal observation of the model gave rise to the largest sensitivity possible in the out-of-plane direction.

Since $v$ is the largest displacement component, it was felt that the angle subtended by the photographic plates in the vertical direction was large enough to obtain accurate readings.

Holograms were made using $10-\mathrm{cm} \times 13-\mathrm{cm}$ Agfa Gevaert 1075 plates. Reconstructions were obtained using a $35-\mathrm{mm}$ reflex camera. Each hologram was masked with a cardboard cutout and observations of the fringe patterns were carried out through fourteen 6.35 -mm diameter holes; eight in hologram 1 and six in hologram 2.

Typical fringe patterns are shown in Fig. 4. Although the disk appeared elliptical in shape in the oblique view, its circular shape was optically restored.

With a microdensitometer to locate fringes and reference lines, eight equidistant scans were made on each of the fourteen views. A magnification of ten

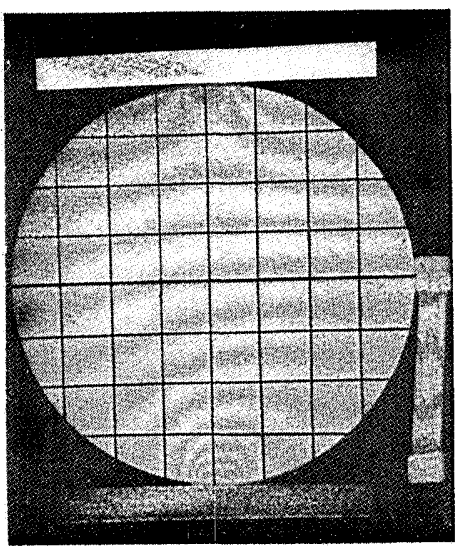

Hologram \#1

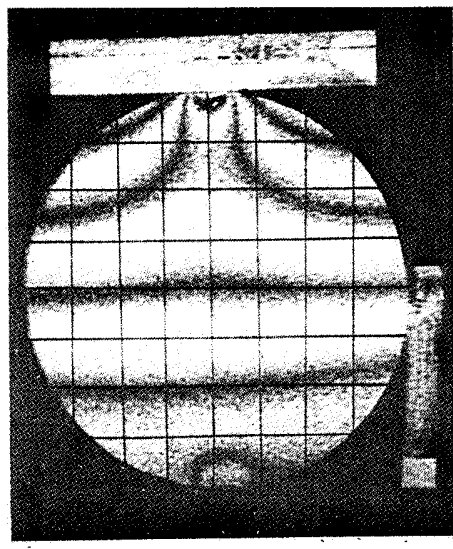

Fig. 4. Typical fringe patterns. 


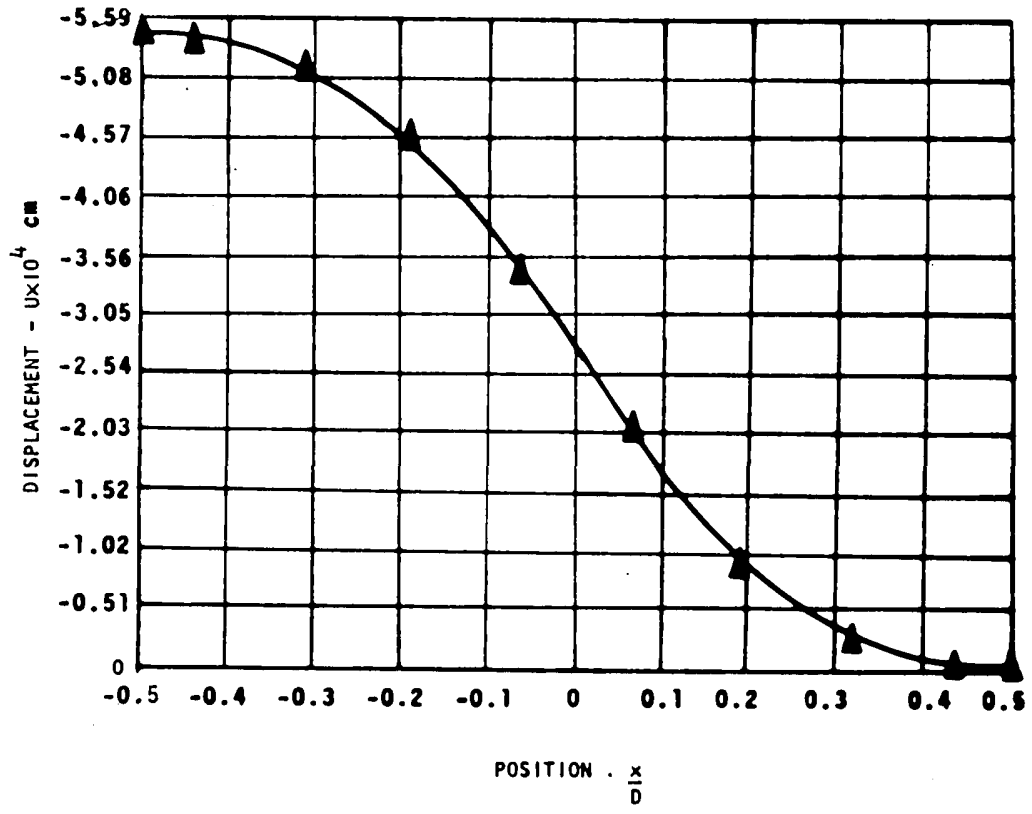

Fig. 5. $u$ displacement along the horizontal diameter.

times was used, and since $1 \mathrm{~mm}$ could be read from the microdensitometer graph paper, distances were measured to $0.1 \mathrm{~mm}$. Fringe order vs distance was plotted for each scan and a scale was chosen so that fringe orders could be read to 0.02 of a fringe. Data were fed to a computer program, designed to reduce the fourteen equations obtained from the two holograms to a $3 \times 3$ system by means of the leastsquares method.

\section{Results}

The $u$ displacement along the horizontal diameter of the disk is given in Fig. 5; the $v$ and $w$ displacements for the central region of the disk are plotted in Fig. 6. Figure 7 shows a pictorial representation of the general motion of the disk under the applied load. The upper platform of the loading frame remains stationary while the lower platform compresses the disk. The point of contact of the disk with the upper loading platform practically did not move and the rigid body motion of the disk can be reduced to three infintesimal rotations around the coordinate axes. Since all the points lying along the horizontal center line are theoretically displaced the same amount in the $y$-direction, it is evident from the $v$ plot of Fig. 5 that a rotation of $\theta_{z}=2.28 \times 10^{-6} \mathrm{rad}$ counterclockwise occurred about the $z$ axis. A study of the $w$ displacement shows that the lower loading point of the disk moved forward through an infinitesimal clockwise rotation of $\theta_{x}=2.18 \times 10^{-5} \mathrm{rad}$ about the $x$ axis. Also, since the $w$ displacements of the two end points of horizontal diameter are different, a rotation of this line in the amount $\theta_{y}=1.4 \times$ $10^{-6}$ rad clockwise occurred about the $y$ axis.

Rather than using the displacements, which contain arbitrary rigid body motion, we will compare strain to evaluate the experimental results. Since the rotations are infinitesimal, their effect on the strains is negligible. Figure 8 shows the theoretical and the experimental $\epsilon_{x}$ and $\epsilon_{y}$. A very good agreement is observed. From the $\epsilon_{x}$ and $\epsilon_{y}$ curves we can compute the $w$ displacement, since it is related by the simple relationship

$$
w=-\nu(t / 2)\left(\epsilon_{x}+\epsilon_{y}\right) .
$$

If we subtract the rigid body motion from the values of $w$, we obtain curve 6 in Fig. 8. Taking the experimental values of $\epsilon_{x}$ and $\epsilon_{y}$ and replacing them in Eq. (10), we obtain curve 5. One must realize that the $w$ displacement is very small in magnitude when compared to the $u$ displacement, $v$ displacement, and the $w$ displacement including rigid body motions. Although there is a difference in shape between curves 5 and 6 , one can see that the order of magnitude of the displacement is correct. For purposes of comparison, we chose to use the experimental values of $\epsilon_{x}$ and $\epsilon_{y}$ in Eq. (10) as opposed to their theoretical counterparts in order to better determine the $w$ displacement. If the method presented here were accurate to within the range of this displacement, the curves would be identical. These observations lead us to the conclusion that the measured displacements were not accurate to $2.54 \times 10^{-6} \mathrm{~cm}$. Since the wavelength of - the utilized light is approximately $64 \times 10^{-6} \mathrm{~cm}$, this implies that we cannot accurately measure displacements that are the order of $1 / 25$ of the wavelength. In view of the smoothness of the results of the displacement measurements, however, we can say that the sensitivity of the method used here is well within the $2.54 \times$ $10^{-6} \mathrm{~cm}$ range. The accuracy can be estimated to be around $8 \times 10^{-6} \mathrm{~cm}$, roughly one-eighth of the wavelength used. 


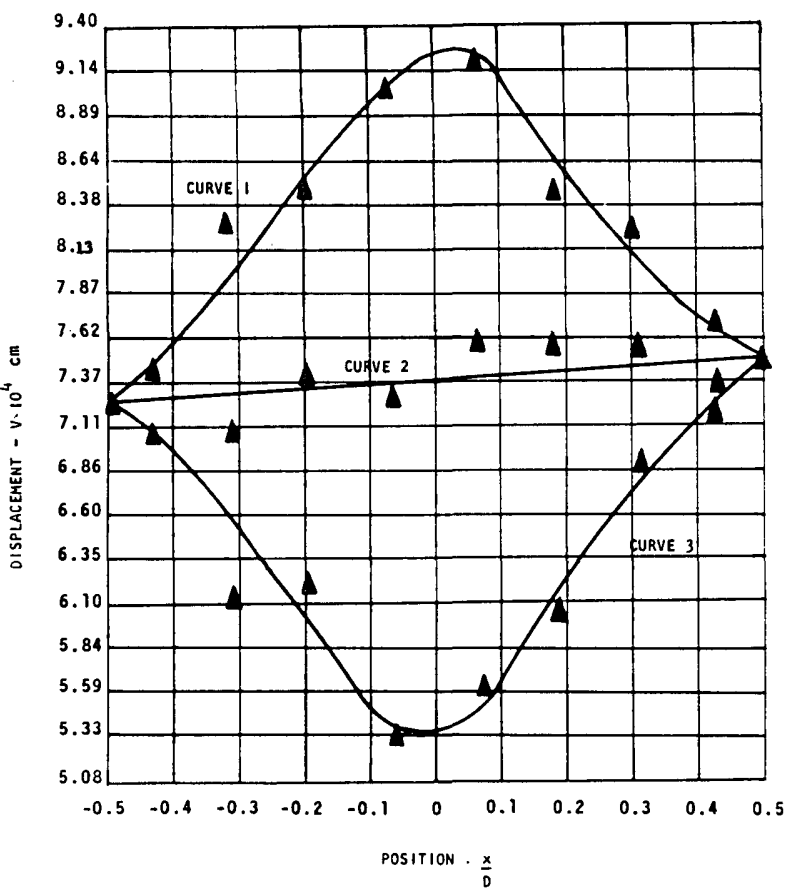

CURVE 1 - V DISP. ALONG POINTS 17-214 (CENTER LINE) CURVE 3 - $V$ DISP. ALONG POINTS $1-8$
Y DISPLACEMENT IN THE $X$ DIRECTIOH

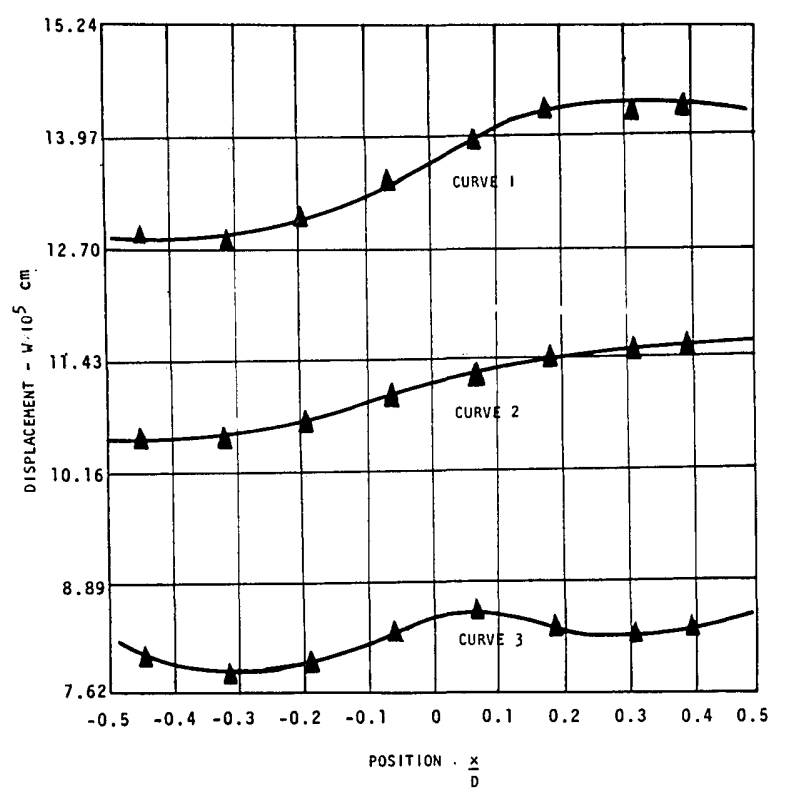

CURVE I - W DISP. ALONG POINTS 17-24 If (CENTER LINE) CURVE 3 - W DISP. ALONG POINTS 1 - 8

W DISPLACEMENT IN THE X DIRECTION

Fig. 6. $v$ and $w$ displacement.

\section{Discussion}

The obtained results show that provided one uses adequate precautions to restrict the amount of random rigid body motions that may be originated by the application of the loads, holographic interferometry can be utilized as a very sensitive and accurate tool of strain analysis.

The introduction of a datum point by connecting the body under analysis and a fixed element provides a simple solution for the use of multiple holograms. In this way, the directions of observation and illumination can be optimized. The ideal situation is to select directions that offer maximum sensitivity for smaller displacement components and minimum sensitivity for the larger components. The use of multiple holograms can be coupled with the use of several points of observation in each hologram, thus providing a means for compensating accidental errors.

We must recall that in the solution of the problem presented in this paper, the fringe location, with respect to the reference system of coordinates, was performed by means of a microdensitometer. Fringe orders were determined by graphical interpolation to $0.01 \lambda$. According to the obtained results, the accuracy of the displacements was around $8 \times 10^{-6} \mathrm{~cm}$, that is, about $1 / 8 \lambda$. It is interesting to compare the above quantity with the results presented by the authors of Ref. 20. Although no specific reference is made to the procedure to locate fringes, it appears from the text of the paper that it was done by eye. Of course, the pattern analyzed in Ref. 20 is very simple. The holographic pattern consists of straight parallel fringes and the analysis was performed on only one line rather than an entire area. Fringe order along the line of interest is very close to linear.

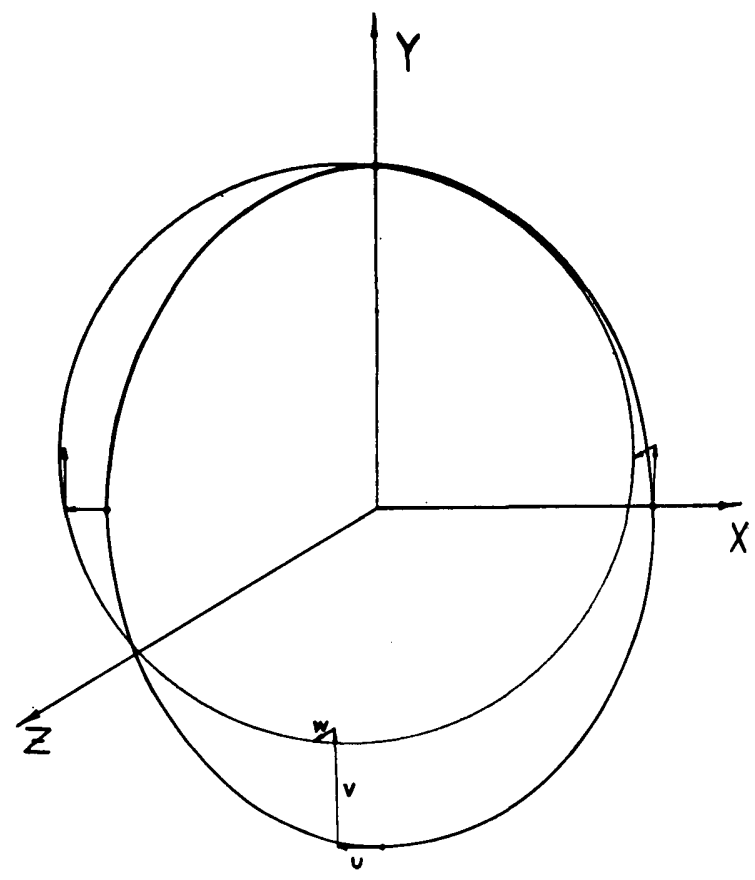

Fig. 7. Motion of the disk. 


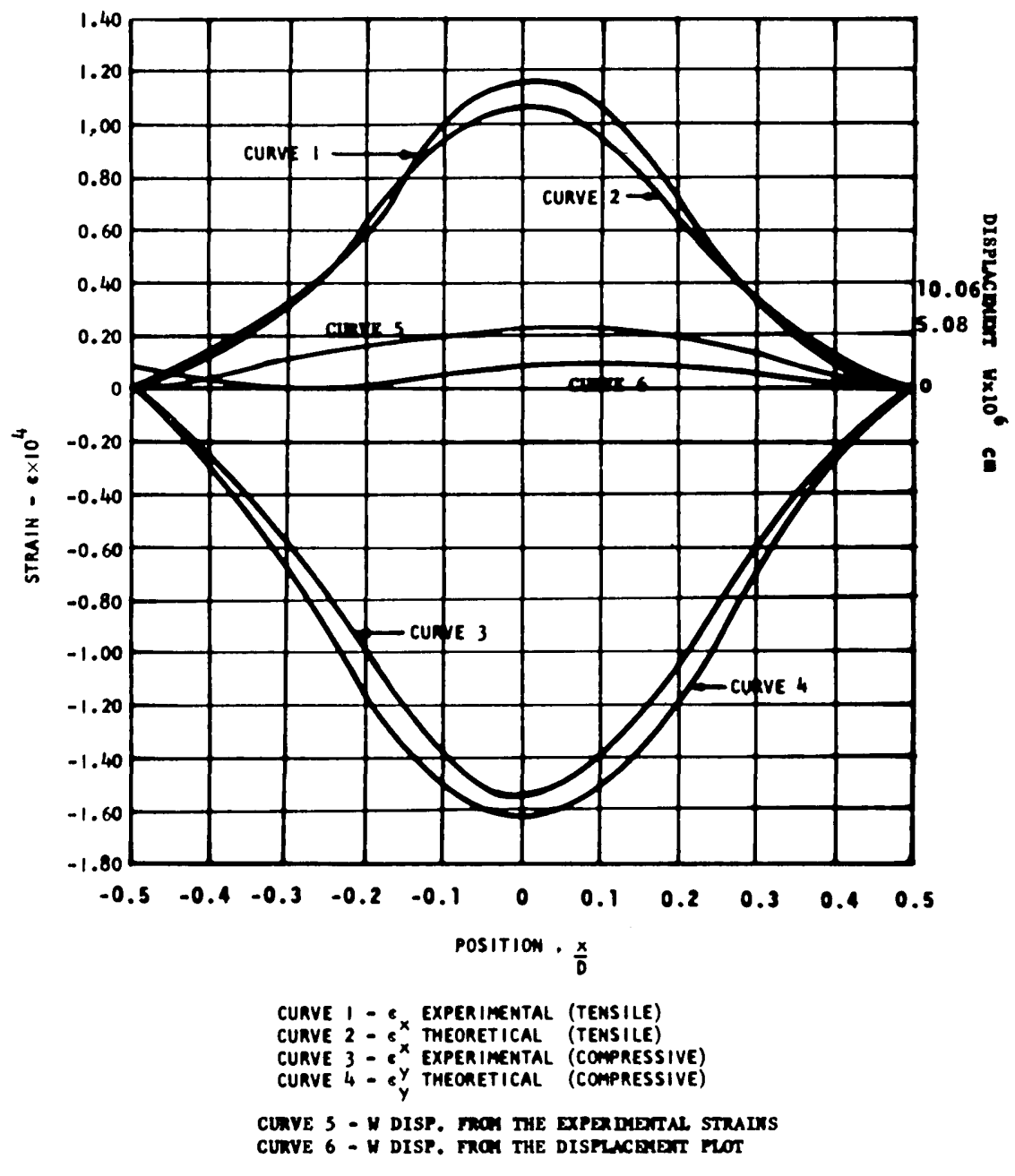

Fig. 8. Theoretical and experimental strains along the horizontal diameter.

From an analysis of the measurements of the deflection of the cantilever beam used as a model and the comparison with theoretical values, the accuracy of the measurements can be found to be $1 / 12 \lambda$. For the other two components, no comparison with theoretical values is given, but values obtained by a timeaverage method are given.

The values of the displacement in the $z$ direction, the direction perpendicular to the longitudinal center line, appear to be random, since according to the symmetry of the model and the type of loading they should be zero. The $v$ values, displacements along the longitudinal center line in the top of the beam, also appear to be random, since they show a change of sign that is not justified by the behavior of a cantilever beam. If we look at Table I of Ref. 20, we can see that the largest value of the components of the displacement vector in the other two reference axes is of the order of $1 / 15 \lambda$. That is, they are smaller than the accuracy achieved for the largest component. This means that the fringe shift in the three holograms taken by the authors was not sufficient to detect quantities of the order of magnitude that they wanted to find.

\section{References}

1. R. L. Powell and K A. Stetson, J. Opt. Soc. Am. 55, 1694 (1965).

2. K. A. Stetson and R. L. Powell, J. Opc. Soc. Am. 56, 1161 (1966).

3. K. A. Haines and B. P. Hildebrand, Appl. Opt. 5, 595 (1966).

4. F. B. Aleksandrov and A. M. Bonch-Bruevich, Tech. Phys. Acad. Sci. USSR 12, 258 (1967).

5. A. E. Ennos, J. Sci. Instrum. (J. Phys. E.) Ser. 2 1, 731 (1968).

6. J. E. Sollid, Appl. Opt. 8, 1587 (1969).

7. R. C. Sampson, Exptl. Mech. 10, 313 (1970).

8. K. A. Stetson, Optik 29, 386 (1969).

9. K. A. Stetson, Optik 31, 576 (1970).

10. K. A. Stetson, J. Opt. Soc. Am. 60, 1378 (1970).

11. S. Walles, Ark. Phys. 40, 299 (1969).

12. J. Ch. Vienot et al. The Engineering Uses of Holography (Cambridge U.P., London 1970), pp. 133-150.

13. C. Froehly et al. Opt. Acta 16, 343 (1969).

14. N. Abramson, Appl. Opt. 8, 1235 (1969).

15. N. Abramson, Appl. Opt. 9, 97 (1970).

16. N. Abramson, 9, Appl. Opt. 2311 (1970).

17. N. Abramson, Appl. Opt. 10, 2155 (1971)

18. A. D. Wilson, Appl. Opt. 9, 2093 (1970).

19. A. D. Wilson, Appl. Opt. 10, 908 (1971).

20. K. Shibayama and H. Uchiyama, Appl. Opt. 10, 2150 (1971).

21. S. K. Dhir and J. P. Sikora, Exp. Mech. 12, 323 (1972). 